\title{
Comparison of evolutionary rates of the regions and nucleotide substitutions in the Allium plastomes
}

\author{
D.O. Omelchenko ${ }^{1,2 *}$, A.A. Krinitsina ${ }^{1,2}$, M.D. Logacheva ${ }^{1,2}$, M.S. Belenikin ${ }^{3}$, \\ E.A. Konorov ${ }^{1,4}$, S.V. Kuptsov ${ }^{1}$, A.P. Seregin ${ }^{1}$, A.S. Speranskaya ${ }^{1,2}$ \\ ${ }^{1}$ Lomonosov Moscow State University, Moscow, Russia \\ ${ }^{2}$ Skolkovo Institute of Science and Technology, Moscow Region, Russia \\ ${ }^{3}$ Moscow Institute of Physics and Technology, Moscow Region, Dolgoprudny, Russia \\ ${ }^{4}$ Vavilov Institute of General Genetics, Moscow, Russia \\ *e-mail:
}

Key words: Allium, evolution, high-throughput sequencing, chloroplast genome

Motivation and Aim: Genus Allium includes several economically important species, some of them of the oldest cultivated crops, along with some rare and endemic species. Still, complete sequences of Allium genomes are majorly underrepresented (only A. cepa, A. sativum and A. obliquum has complete chloroplast DNA records in GenBank). Modern molecular taxonomy of the genus is based on nuclear internal transcribed spacers (ITS) sequences analysis. Here we performed sequencing, assembly and comparative analysis on evolution and diversity of the complete sequences of the chloroplast genomes of Allium species, representing all three evolutionary lines of this genus.

Methods and Algorithms: The plastomes of 11 Allium species (A. ursinum, A. paradoxum, A. zebdanense, A. victorialis, A. macleanii, A. fistulosum, A. nutans, A. platyspathum, A. obliquum, A. shoenoprasum, A. pskemense) were sequenced on the Illumina MiSeq and assembled de novo (using various assemblers, including Velvet and MIRA4). Sequences were annotated by DOGMA and GeSeq, followed by manual review and correction of annotated features where necessary. Phylogenetic analysis was performed with Mr. Bayes and phangorn R package. Length of branches was calculated, and natural selection was detected by PAML and HyPhy. Evolutionary rates were calculated using Erable.

Results: Besides expected differences represented by small indels in intergenic spacers, there are conspicuous characteristic large deletions and nonsynonymous substitutions in some genes in the Allium plastomes, some of them specific to the evolutionary lines. A. paradoxum plastome showed most interesting results that differ it from other Allium species - 4.9 kbp inversion of rpl32-ndhE region with impairment of $n d h G$ and $n d h F$ genes in SSC. Evolutionary analysis showed that some genes commonly used for phylogenetic analysis (e.g., matK) have less constant evolutionary rate within the genus Allium compared to intergenic regions.

Conclusion: Analysis of Allium plastomes revealed prominent species specific and even evolutionary line specific differences in sequence and gene set. We assume the effect of evolution on various parts of Allium plastomes differs in character between species of this genus.

Acknowledgements: Supported by the RFBR grant No. 18-04-01203. 